\title{
Toenail selenium levels and prevalence of dyslipidaemia among Korean adults
}

\author{
Jiyoung Jang ${ }^{1}$, J. Steven Morris ${ }^{2,3}$ and Kyong Park ${ }^{1 *}$ \\ ${ }^{1}$ Department of Food and Nutrition, Yeungnam University, Gyeongsan, Gyeongbuk 38541, Republic of Korea \\ ${ }^{2}$ Department of Research and Education, University of Missouri Research Reactor, Columbia, MO 65211, USA \\ ${ }^{3}$ Department of Research Services, Harry S. Truman Memorial Veterans Hospital, Columbia, MO 65205, USA
}

(Submitted 27 April 2017 - Final revision received 26 July 2017 - Accepted 7 August 2017)

\begin{abstract}
Multiple studies have elucidated the antioxidant properties of Se, which are now well known among the nutrition and biomedical science communities. Recently, considerable interest has been focused on the possible association between Se exposure and risk of metabolic disease, such as lipid dysregulation; however, there is limited epidemiological data on this topic. The present study aimed to investigate associations between toenail Se levels and dyslipidaemia or individual lipid levels, and to examine the effect of dietary supplement use on these associations. We analysed baseline data from a cohort in the Yeungnam area, including 232 men and 269 women. Information on demographic, dietary and lifestyle characteristics was obtained through a self-reported questionnaire. Se levels in toenail specimens were measured using neutron activation analysis. Fasting blood lipid levels were measured during medical examinations. After adjusting for multiple confounding variables, we observed no association between toenail Se levels and dyslipidaemia or individual lipid profiles. However, the association was modified by dietary supplement use. Among the supplement users, higher toenail Se levels were associated with a higher prevalence of lipid dysregulation, whereas non-users exhibited a lower prevalence of lipid dysregulation. Associations between toenail Se levels, lipid levels and dyslipidaemia may be influenced by taking dietary supplements. Future large-scale, prospective cohort studies should be conducted to further evaluate the association between Se levels in the body and metabolic health effects in light of increasing rates of dietary supplement use.
\end{abstract}

Key words: Toenail analysis: Selenium: Dyslipidaemia: Epidemiological effect modifiers: Dietary supplements: Asian adults

Se is an essential mineral in the human body, and acts as an important component of antioxidant enzymes, such as glutathione peroxidase ${ }^{(1,2)}$. Enzymes containing Se are referred to as selenoproteins; to date, at least thirty mammalian selenoproteins have been identified, of which approximately twenty-five are present in humans ${ }^{(3)}$.

Previous studies have reported that cereal ${ }^{(2,4-6)}$, meat ${ }^{(1,2,6,7)}$, fish and other seafood ${ }^{(2,6,8,9)}$ and some fruit and vegetables ${ }^{(2,6)}$ are major dietary sources of Se. However, the Se content of crops is greatly dependent on the soil in which they are grown $^{(1,10,11)}$. In countries with low soil-Se levels, the overall dietary Se levels obtained from consumption of crops grown in the soil may be minimal. Se deficiency may decrease antioxidant activity and cause serious health problems. One example is the Keshan disease ${ }^{(2,12)}$, which was first found in Keshan County of Heilongjiang province, Northeast China, where the soil Se content is extremely low ${ }^{(12,13)}$. Se supplementation may be an option to increase Se levels in populations residing in regions with low soil-Se levels; however, the dosage, reference range for intake and safety issues for Se supplementation have not been completely established ${ }^{(1)}$.
Experimental studies have shown that the antioxidant effects of Se are involved in the prevention of lipid oxidation, thereby preventing the development of dyslipidaemia and progression from dyslipidaemia to CVD or cancer ${ }^{(14-16)}$. However, epidemiological studies are limited and have mostly been conducted in Europe and the USA ${ }^{(17-19)}$. In addition, the reference range for Se, which is based on a biomarker of long-term exposure to Se, remains unclear ${ }^{(20)}$. Moreover, the health effects of Se may vary depending on the genetic variants of selenoproteins ${ }^{(21,22)}$, dietary sources of Se (e.g. foods or dietary supplements) ${ }^{(23)}$ or the presence of effect-modifiers ${ }^{(24,25)}$.

A recent epidemiological study of residents in the Yeungnam area in South Korea examining the association between toenail $\mathrm{Hg}$ levels and dyslipidaemia found that toenail Se status is an effect-modifier of this association ${ }^{(24)}$. However, it is unclear whether Se levels in the body directly contribute to dyslipidaemia in this population; investigation of this association might be even more relevant given the high consumption of fish rich in Se by residents in the Yeungnam area. It is also necessary to determine the optimal Se reference range for the prevention of chronic diseases, particularly lipid dysregulation.

Abbreviations: SELEN, Trace Element Study of Korean Adults in Yeungnam Area; TC, total cholesterol.

* Corresponding author: K. Park, fax +82 53810 4768, email kypark@ynu.ac.kr 
The present study aimed to determine the association between toenail Se levels and dyslipidaemia, and to identify potential effect-modifiers of this association among Korean adults.

\section{Methods \\ Study population}

This study was conducted using baseline data from the Trace Element Study of Korean Adults in Yeungnam Area (SELEN) cohort study involving adults aged $\geq 35$ years in the Yeungnam area of South Korea. Baseline participant data, including demographic, lifestyle and dietary information, were collected using a self-report questionnaire developed by SELEN investigators. The complete data on demographic, lifestyle, dietary and health information and toenail Se levels for 501 SELEN study participants were assessed for the final analysis.

Informed consent was obtained from all participants. The present study was approved by the institutional review board (IRB) at Yeungnam University Medical Center in compliance with the ethical standards for research, and was conducted with consideration of the safety and rights of the participants (IRB no. YUH-12-0468-O94).

\section{Measurements}

Participants were categorised into two groups on the basis of their education levels: high school graduates or below, and university graduates or above. On the basis of the alcohol consumption status, participants were categorised as drinkers or non-drinkers. Similarly, on the basis of the monthly household income, participants were categorised into quintiles. In terms of smoking status, patients were categorised as current smokers, former smokers and non-smokers. Physical activity frequency (per week) and hours (per day) were examined, and metabolic equivalent tasks (MET-h/week) were calculated in accordance with physical activity intensity ${ }^{(26)}$.

Information on participants' anthropometric measurements and blood lipid levels was collected from health examinations conducted by the Korea National Health Insurance Service (KNHIS). All Korean workers and their dependants are legally required to have biennial health examinations through the KNHIS. We asked participants to undergo a health examination at least 6 months after completing the baseline questionnaire and toenail sample collection, and send a copy of their health examination results to the SELEN researchers. According to the KNHIS guidelines, weight, height and waist circumference (WC) measurements, as well as blood samples, were collected by trained nurses in the relevant medical institution where the medical examination was conducted ${ }^{(27)}$. We calculated the BMI of each participant, using the weight and height values recorded as part of the medical examination and by dividing the weight in kilograms by the square of height in metres. At the health examination, participants provided laboratory specimens after fasting for at least $12 \mathrm{~h}$. Serum lipid levels, including TAG, total cholesterol (TC), LDL-cholesterol and HDL-cholesterol levels were measured in clinical laboratories by staff with certified qualifications in accordance with strict management protocols of the KNHIS. Atherogenic index of plasma (AIP) was calculated according to the formula $\log (\mathrm{TAG} / \mathrm{HDL}-$ cholesterol) ${ }^{(28)}$. The criteria for dyslipidaemia and related health conditions used in the present study were in accordance with the National Cholesterol Education Program Adult Treatment Panel III criteria ${ }^{(29)}$. Dyslipidaemia was confirmed if one or more of the following four criteria were met: (1) hypercholesterolaemia, defined as serum TC level $\geq 6.22 \mathrm{mmol} / \mathrm{l}$ or taking lipid-lowering drugs; (2) hyper-LDL-cholesterolaemia, defined as serum LDL-cholesterol level $\geq 4.14 \mathrm{mmol} / \mathrm{l}$ or taking lipidlowering drugs; (3) hypertriacylglycerolaemia, defined as serum TAG level $\geq 2 \cdot 26 \mathrm{mmol} / \mathrm{l}$; and (4) hypo-HDL-cholesterolaemia, defined as serum HDL-cholesterol level $\leq 1.04 \mathrm{mmol} / 1$ for men and serum HDL-cholesterol level $\leq 1.30 \mathrm{mmol} / 1$ for women.

Dietary information was obtained through a validated 146-item semiquantitative FFQ, specifically developed for the SELEN participants ${ }^{(30)}$. In particular, for this study, we focused on a subset of questions designed to obtain information on the use of dietary supplements. Specifically, the participants were asked to report whether they currently used any dietary supplements. If this response was affirmative, participants were then asked to report the following: type of dietary supplement(s) taken (e.g. multivitamins and minerals), the brand name, dosage per day and duration of dietary supplement use. For this study, dietary supplement use was defined as consumption of at least one type of dietary supplement per day.

Although blood Se level has been used as a major biomarker in many studies, it represents only short-term Se exposure ${ }^{(31)}$. Se is stored in multiple locations, including blood, hair and nails. Among these storage sites, toenails are more likely to represent long-term exposure to Se than other storage sites $^{(31-33)}$. We provided participants the sample-collection protocol for collecting nail specimens for trace-element analysis. Participants were asked to remove nail polish, and clean and dry their toes before cutting toenails. They were also asked to uniformly cut toenails from all ten toes, seal them in an envelope and send them to the SELEN researchers via postal mail. After the collection of toenail samples was completed, researchers conducted visual inspection of the participants' nail clippings to detect whether the samples were contaminated by nail polish or nail colour containing garden balsam. They also measured the sample weights to confirm that the nail specimens met the minimum analysable weight requirement. The toenail clippings were then sent to the University of Missouri Research Reactor Center in Missouri, USA, to quantify toenail Se levels using neutron activation analysis. Details on the validity of the analytical methods and measurement tools used in this study have been described previously ${ }^{(31,34-36)}$. In brief, each sample (8-90 mg) was cleaned ultrasonically with dilute nitric acid and deionised water before analysis, placed in a precleaned, highdensity polyethylene vial $(0.4 \mathrm{ml})$ and subjected to neutron irradiation at a thermal neutron flux of $6.5 \times 10^{13} \mathrm{n} / \mathrm{cm}^{2}$ per $\mathrm{s}$. During this irradiation, $\mathrm{Se}^{77 \mathrm{~m}}$ is produced, which decays by isomeric transition with a half-life of $17.36 \mathrm{~s}$, producing a gamma ray with energy of $161.9 \mathrm{keV}$ that is quantified by high-resolution gamma-ray spectroscopy. The spectrometer consists of a high-purity germanium detector, power supply, spectroscopy amplifier, analog-to-digital convertor and loss-free 
counting module provided by ORTEC operating on a Canberra Genie gamma-ray spectroscopy network (Canberra). Toenail Se concentrations were determined by standard comparison using a certified Se standard solution (High-Purity Standards) and traceable to the US National Institutes of Standards and Technology (NIST SRM 3149; Gaithersburg, MD). All analytical procedures were performed by trained laboratory staff blinded to person-identifiable information, demographic descriptors and nutritional metrics. For approximately $40 \%$ of the subjects, there was adequate sample to prepare and analyse in duplicate. In those cases, the mean percent $\mathrm{CV}$ (CV) for all duplicate pairs was $2 \cdot 14 \%$ and the range was $0.09 \%$ to $8.47 \%$. For each analysis, batch replicates of a biological standard reference material, issued by the US NIST (NIST SRM 1577, Bovine Liver; NIST), having a certified Se concentration, were analysed by the same methodology as described for the toenail samples. The average Se concentration measured in these replicates (1.136 (SD 0.016$) \mu \mathrm{g} / \mathrm{g}, \mathrm{CV}: 1.44 \%$ ) was in good agreement with the certified Se concentration $(1 \cdot 1(\mathrm{sD} 0 \cdot 1) \mu \mathrm{g} / \mathrm{g})$.

\section{Statistical analysis}

We conducted a sample size calculation using G*Power software(version 3.1.9.2; University of Kiel) with an $\alpha$-error probability of $0 \cdot 05$, power (1- $\beta$ error probability) of 0.95 and effect size (OR) of $1 \cdot 24^{(37)}$. On the basis of the result of this calculation, the total sample size needed was at least 375. Assuming that $30 \%$ of the data collected would be missing in information owing to participant attrition and/or measurement errors, we determined the necessary total sample size to be approximately 500 participants. To compare various demographic, lifestyle, dietary and health factors with Se levels, the cohort was divided into tertiles according to toenail Se levels. Categorical variables were compared using the $\chi^{2}$ test, and mean values were compared with continuous variables using ANOVA and the Tukey's post hoc test. Potential confounding variables were selected based on previous studies and preliminary analysis. OR and 95\% CI were obtained using multivariable logistic regression to analyse the prevalence of dyslipidaemia and individual lipid profiles, according to tertiles of toenail Se levels. To control the effect of potential confounders, the following models were tested: model $1=$ adjusted for age and sex; model $2=$ model 1 plus adjusted for education level, household income, smoking status, BMI and total energy intake; and model $3=$ model 2 plus further adjusted for MET, alcohol intake, fat intake, toenail $\mathrm{Hg}$ level and family history of chronic diseases (hypertension, diabetes, CVD and cancer). Multivariable adjusted mean serum lipid levels, including TC, HDL-cholesterol, LDL-cholesterol and TAG, were calculated using general linear regression analysis. Potential effect-modifiers, including various demographic, lifestyle, dietary and health-related variables, were examined using multiplicative terms in logistic regression. As the use of dietary supplements was an effect-modifier of the association between toenail Se levels and dyslipidaemia, we further examined the association of Se with dyslipidaemia and individual lipid profiles stratified by the use of dietary supplements. The $P$ value for trend in tertiles of Se levels in linear regression was evaluated using the median value of the category as a continuous variable.
All analyses in the present study were performed using SAS (version 9.3; SAS Institute), and $P<0.05$ was considered statistically significant.

\section{Results}

In total, 501 participants were divided into groups according to tertiles of toenail Se levels, and their general characteristics were compared (Table 1 ). The mean values of toenail Se levels were $0.60 \mu \mathrm{g} / \mathrm{g}$ in the 1 st tertile, $0.69 \mu \mathrm{g} / \mathrm{g}$ in the 2 nd tertile and $0.79 \mu \mathrm{g} / \mathrm{g}$ in the $3 \mathrm{rd}$ tertile. The average age of the study participants was approximately 44 years. We observed higher toenail Se levels among younger participants than in those from older participants $(P=0.002)$. Seventy-nine percent of all participants consumed alcohol, and the proportion of drinkers was higher among those with higher levels of toenail Se $(P=0 \cdot 02)$. No significant differences in the multivariate-adjusted mean serum lipid levels were observed among the tertiles.

General characteristics of participants were compared in accordance with the use or non-use of dietary supplements, and are shown in Table 2 . Dietary supplement users were more likely to be female $(P=0 \cdot 008)$, non-smokers $(P<0 \cdot 001)$, college graduates or above $(P=0.004)$ and have a family history of hypertension $(P=0.03)$ or diabetes $(P=0.03)$. Furthermore, those with a higher household income were more likely to use dietary supplements $(P=0.008)$. After adjusting for multiple demographic and lifestyle variables, no significant differences were observed in serum TC, LDL-cholesterol, HDL-cholesterol and AIP between dietary supplement users and non-users.

The OR of dyslipidaemia and lipid abnormalities according to tertiles of toenail Se are shown in Table 3. In minimally and fully adjusted models, toenail Se levels were not associated with the prevalence of dyslipidaemia, hypercholesterolaemia, hypo-HDL-cholesterolaemia, hyper-LDL-cholesterolaemia and hypertriacylglycerolaemia.

However, we observed that the associations between toenail Se level and dyslipidaemia or individual lipid profiles were modified by the use or non-use of dietary supplements (Table 4). Among dietary supplement users, the prevalence of hypercholesterolaemia was 3.56 times higher in participants in the 3rd tertile of toenail Se levels than in those from the 1 st tertile (OR 3.56; 95\% CI 1.12, 11.26); the prevalence of hypertriacylglycerolaemia was 2.68 times higher in participants in the 3 rd tertile than in those from the 1st tertile (OR 2.68; $95 \% \mathrm{CI}$ $1.09,6.64)$; and the prevalence of dyslipidaemia was 2.72 times higher in participants in the 3rd tertile than in the those in the 1st tertile (OR $2 \cdot 72 ; 95 \%$ CI $1 \cdot 41,5 \cdot 26$ ). Conversely, for nonusers of dietary supplements, these associations were reversed, showing an OR of 0.21 (95\% CI $0.07,0.59)$ for hypertriacylglycerolaemia and an OR of 0.40 (95\% CI $0 \cdot 17,0 \cdot 91)$ for dyslipidaemia.

\section{Discussion}

The present study examined the associations between toenail Se levels and prevalence of dyslipidaemia and individual lipid profiles using baseline data from the SELEN cohort study. We found that these associations were modified by dietary 
Table 1. Demographic and lifestyle characteristics of the study participants according to the tertiles $(T)$ of toenail selenium levels (Numbers and percentages; mean values with their standard errors)

\begin{tabular}{|c|c|c|c|c|c|c|c|}
\hline \multirow[b]{3}{*}{ Characteristics } & \multicolumn{6}{|c|}{ Tertile of toenail Se $(\mu \mathrm{g} / \mathrm{g})$} & \multirow[b]{3}{*}{$P^{*}$} \\
\hline & \multicolumn{2}{|c|}{$\mathrm{T} 1(n$ 167) $(0.48-0.66)$} & \multicolumn{2}{|c|}{ T2 $(n$ 167) $(0.66-0.71)$} & \multicolumn{2}{|c|}{ T3 $(n$ 167) $(0.71-1 \cdot 10)$} & \\
\hline & $n$ & $\%$ & $n$ & $\%$ & $n$ & $\%$ & \\
\hline Sex & & & & & & & 0.7 \\
\hline Men & 78 & $46 \cdot 71$ & 81 & 48.50 & 73 & $43 \cdot 71$ & \\
\hline Women & 89 & $53 \cdot 29$ & 86 & 51.50 & 94 & $56 \cdot 29$ & \\
\hline $\mathrm{BMI}$ & & & & & & & 0.4 \\
\hline Underweight & 5 & 3.01 & 5 & 2.99 & 3 & 1.80 & \\
\hline Normal & 79 & 47.59 & 73 & 43.71 & 79 & 47.31 & \\
\hline Overweight & 52 & 31.33 & 47 & $28 \cdot 14$ & 39 & 23.35 & \\
\hline Obese & 30 & 18.07 & 42 & $25 \cdot 15$ & 46 & 27.54 & \\
\hline Smoking status & & & & & & & 0.3 \\
\hline Non-smoker & 104 & $62 \cdot 28$ & 104 & $62 \cdot 28$ & 119 & 71.26 & \\
\hline Former smoker & 26 & 15.57 & 31 & 18.56 & 23 & 13.77 & \\
\hline Current smoker & 37 & $22 \cdot 16$ & 32 & $19 \cdot 16$ & 25 & 14.97 & \\
\hline Alcohol consumption & & & & & & & 0.02 \\
\hline Non-drinker & 48 & 28.74 & 28 & $16 \cdot 77$ & 31 & 18.56 & \\
\hline Drinker & 119 & $71 \cdot 26$ & 139 & $83 \cdot 23$ & 136 & 81.44 & \\
\hline Education & & & & & & & 0.01 \\
\hline High school graduate or lower & 69 & 41.32 & 43 & $25 \cdot 90$ & 50 & 29.94 & \\
\hline College graduate or higher & 98 & 58.68 & 123 & $74 \cdot 10$ & 117 & 70.06 & \\
\hline Monthly household income (KRW) & & & & & & & 0.5 \\
\hline$<3000000$ & 44 & $26 \cdot 35$ & 30 & 17.96 & 34 & $20 \cdot 36$ & \\
\hline $3-3990000$ & 36 & 21.56 & 49 & $29 \cdot 34$ & 37 & $22 \cdot 16$ & \\
\hline $4-4990000$ & 29 & 17.37 & 27 & $16 \cdot 17$ & 33 & 19.76 & \\
\hline $5-5990000$ & 26 & 15.57 & 22 & $13 \cdot 17$ & 28 & $16 \cdot 77$ & \\
\hline$\geq 6000000$ & 32 & $19 \cdot 16$ & 39 & 23.35 & 35 & 20.96 & \\
\hline Residence area & & & & & & & 0.5 \\
\hline Urban & 91 & 54.49 & 101 & $60 \cdot 48$ & 96 & 57.49 & \\
\hline Rural & 76 & $45 \cdot 51$ & 66 & 39.52 & 71 & 42.51 & \\
\hline Family history of hypertension & 53 & 31.90 & 53 & 31.90 & 55 & 33.10 & 0.9 \\
\hline Family history of diabetes & 31 & $18 \cdot 80$ & 37 & $22 \cdot 20$ & 42 & $25 \cdot 50$ & 0.3 \\
\hline \multirow[t]{2}{*}{ Dietary supplement use } & 92 & 55.09 & 91 & 54.49 & 101 & $60 \cdot 48$ & 0.5 \\
\hline & Mean & SE & Mean & $\mathrm{SE}$ & Mean & $\mathrm{SE}$ & \\
\hline Age (years) & $46 \cdot 00^{\mathrm{a}}$ & 0.41 & $44 \cdot 40^{b}$ & 0.41 & $44 \cdot 10^{c}$ & 0.41 & 0.002 \\
\hline Toenail Se $(\mu \mathrm{g} / \mathrm{g})$ & $0.60^{\mathrm{a}}$ & 0.003 & $0.69^{b}$ & 0.003 & $0.79^{c}$ & 0.003 & $<0.001$ \\
\hline Toenail $\mathrm{Hg}(\mu \mathrm{g} / \mathrm{g})$ & 0.38 & 0.02 & 0.42 & 0.02 & 0.42 & 0.02 & 0.2 \\
\hline MET (h/week) & 33.98 & 3.64 & 33.76 & 3.64 & 31.47 & 3.66 & 0.9 \\
\hline Total fat intake $(\mathrm{g} / \mathrm{d}) \dagger$ & 39.24 & 0.80 & 39.95 & 0.80 & 41.06 & 0.80 & 0.3 \\
\hline \multicolumn{8}{|l|}{ Blood lipid levels $\ddagger$} \\
\hline $\mathrm{TAG}(\mathrm{mmol} / \mathrm{l})$ & 1.30 & 0.07 & 1.34 & 0.07 & 1.34 & 0.08 & 0.6 \\
\hline Total cholesterol $(\mathrm{mmol} / \mathrm{l})$ & 4.64 & 0.22 & 4.90 & 0.24 & 4.68 & 0.24 & 0.9 \\
\hline HDL-cholesterol (mmol/l) & 1.40 & 0.06 & 1.44 & 0.06 & 1.47 & 0.06 & 0.3 \\
\hline LDL-cholesterol $(\mathrm{mmol} / \mathrm{l})$ & 2.99 & 0.07 & 2.97 & 0.08 & 2.98 & 0.08 & 0.9 \\
\hline
\end{tabular}

supplement use. Among supplement users, those with higher Se levels were more likely to have prevalent dyslipidaemia and hypertriacylglycerolaemia than those with lower Se levels. However, among supplement non-users, those with higher Se levels were more likely to have lower rates of dyslipidaemia and hypertriacylglycerolaemia than those with lower Se levels.

Considering that Se content in foods varies according to the Se levels in the soil in which the crops were grown ${ }^{(6)}$, Se intake and, consequently, the level of Se in the body is likely dependent on an individual's place of residence. The Nurses' Health and Health Professional studies in the USA have shown that average concentrations of toenail Se were 0.77 (SD 0.13) $\mu \mathrm{g} / \mathrm{g}$ in men and $0.84(\mathrm{SD} 0 \cdot 15) \mu \mathrm{g} / \mathrm{g}$ in women ${ }^{(38)}$. The average concentrations of toenail Se in the Netherlands Cohort Study in Europe were relatively lower at $0.55(\mathrm{SD} 0.13) \mu \mathrm{g} / \mathrm{g}$ in men and $0.57(\mathrm{sD} 0.15) \mu \mathrm{g} / \mathrm{g}$ in women ${ }^{(39)}$. The average concentrations of toenail Se in the SELEN participants were 0.68 (SD 0.08) $\mu \mathrm{g} / \mathrm{g}$ in men and $0.70(\mathrm{SD} 0.09) \mu \mathrm{g} / \mathrm{g}$ in women, which fall in between values reported in the USA and Europe. Considering the U-shaped association between Se levels and metabolic health risks and a relatively narrow safe range of Se levels that is nontoxic $^{(24,40)}$, the study results of the SELEN participants may 
Table 2. Demographic and lifestyle characteristics of the study participants according to the use of dietary supplements

(Numbers and percentages; mean values with their standard errors)

\begin{tabular}{|c|c|c|c|c|c|}
\hline \multirow[b]{3}{*}{ Characteristics } & \multicolumn{4}{|c|}{ Supplement use } & \multirow[b]{3}{*}{$P^{*}$} \\
\hline & \multicolumn{2}{|c|}{ No $(n 217)$} & \multicolumn{2}{|c|}{ Yes $(n 284)$} & \\
\hline & $n$ & $\%$ & $n$ & $\%$ & \\
\hline Sex & & & & & 0.008 \\
\hline Men & 115 & 53.00 & 117 & 41.20 & \\
\hline Women & 102 & $47 \cdot 00$ & 167 & 58.80 & \\
\hline BMI & & & & & 0.2 \\
\hline Underweight & 8 & 3.69 & 6 & 1.77 & \\
\hline Normal & 101 & 46.54 & 130 & 45.94 & \\
\hline Overweight & 52 & 23.96 & 86 & 30.39 & \\
\hline Obese & 56 & $25 \cdot 81$ & 62 & 21.91 & \\
\hline Smoking status & & & & & $<0.001$ \\
\hline Non-smoker & 130 & 59.91 & 197 & 69.37 & \\
\hline Former smoker & 30 & 13.82 & 50 & 17.61 & \\
\hline Current smoker & 57 & $26 \cdot 27$ & 37 & 13.03 & \\
\hline Alcohol consumption & & & & & 0.6 \\
\hline Non-drinker & 44 & $20 \cdot 28$ & 63 & $22 \cdot 18$ & \\
\hline Drinker & 173 & 79.72 & 221 & 77.82 & \\
\hline Education & & & & & 0.004 \\
\hline $\begin{array}{l}\text { High school graduate or } \\
\text { lower }\end{array}$ & 85 & $39 \cdot 35$ & 77 & $27 \cdot 11$ & \\
\hline College graduate or higher & 131 & $60 \cdot 65$ & 207 & 72.89 & \\
\hline $\begin{array}{l}\text { Monthly household income } \\
\text { (KRW) }\end{array}$ & & & & & 0.008 \\
\hline$<3000000$ & 51 & 23.50 & 57 & 20.07 & \\
\hline 3-3990000 & 66 & 30.41 & 56 & 19.72 & \\
\hline $4-4990000$ & 39 & 17.97 & 50 & $17 \cdot 61$ & \\
\hline $5-5990000$ & 24 & 11.06 & 52 & 18.31 & \\
\hline$\geq 6000000$ & 37 & 17.05 & 69 & 24.30 & \\
\hline Residence area & & & & & 0.1 \\
\hline Urban & 115 & 53.00 & 173 & 60.92 & \\
\hline Rural & 102 & 47.00 & 111 & 39.08 & \\
\hline Family history of hypertension & 58 & $26 \cdot 98$ & 103 & $36 \cdot 40$ & 0.03 \\
\hline \multirow[t]{2}{*}{ Family history of diabetes } & 38 & $17 \cdot 59$ & 72 & $25 \cdot 62$ & 0.03 \\
\hline & Mean & SE & Mean & SE & \\
\hline Age (years) & $44 \cdot 10$ & 0.30 & $45 \cdot 30$ & 0.41 & 0.01 \\
\hline Toenail Se $(\mu \mathrm{g} / \mathrm{g})$ & 0.69 & 0.005 & 0.69 & 0.01 & 0.5 \\
\hline Toenail Hg ( $\mu \mathrm{g} / \mathrm{g})$ & 0.41 & 0.01 & 0.40 & 0.02 & 0.7 \\
\hline MET (h/week) & 34.07 & $2 \cdot 62$ & 31.28 & 3.52 & 0.1 \\
\hline Total fat intake $(\mathrm{g} / \mathrm{d}) \dagger$ & 39.59 & 0.57 & 40.99 & 0.78 & 0.5 \\
\hline \multicolumn{6}{|l|}{ Blood lipid levels $\ddagger$} \\
\hline TAG (mmol/l) & $1 \cdot 34$ & 0.07 & 1.30 & 0.06 & 0.6 \\
\hline Total cholesterol $(\mathrm{mmol} / \mathrm{l})$ & 4.70 & 0.21 & 4.75 & 0.20 & 0.8 \\
\hline HDL-cholesterol (mmol/l) & 1.42 & 0.06 & 1.44 & 0.05 & 0.7 \\
\hline LDL-cholesterol (mmol/l) & $2 \cdot 96$ & 0.07 & 3.00 & 0.07 & 0.6 \\
\hline AIP & 0.63 & 0.06 & 0.61 & 0.05 & 0.8 \\
\hline
\end{tabular}

KRW, Korean Republic Won; MET, metabolic equivalent tasks; AIP, atherogenic index of plasma.

${ }^{*} P$ values are derived from general linear regression analysis or $x^{2}$ test.

$\dagger$ Adjusted for total energy intake.

$\ddagger$ Adjusted for age, sex, household income, physical activity level, education level, $\mathrm{BMI}$, total energy intake, alcohol consumption and smoking status.

provide meaningful scientific data for the formulation of dietary guidelines for safe and optimal daily intake of Se.

In this study, we observed that the direction of association between toenail Se levels and lipid dysregulation was reversed in accordance with dietary supplementation. Se is an essential trace element with antioxidant properties; adequate intakes of Se and other antioxidants decrease lipid oxidation and increase protective effects against various metabolic health conditions,
Table 3. Lipid profiles according to the tertiles $(T)$ of toenail selenium levels (Odds ratios and $95 \%$ confidence intervals)

\begin{tabular}{|c|c|c|c|c|c|c|}
\hline \multirow[b]{3}{*}{ Lipid profiles } & \multicolumn{5}{|c|}{ Tertile of toenail Se level $(\mu \mathrm{g} / \mathrm{g})$} & \multirow[b]{3}{*}{$P_{\text {for trend }}$} \\
\hline & \multirow{2}{*}{$\frac{\mathrm{T} 1}{\mathrm{OR}}$} & \multicolumn{2}{|r|}{ T2 } & \multicolumn{2}{|r|}{ T3 } & \\
\hline & & OR & $95 \% \mathrm{Cl}$ & OR & $95 \% \mathrm{Cl}$ & \\
\hline \multicolumn{7}{|c|}{ Hypercholesterolaemia } \\
\hline Cases $(n)$ & 11 & & 21 & & 17 & \\
\hline Model $1^{\star}$ & 1.00 & $2 \cdot 16$ & $1.00,4.68$ & 1.73 & $0.78,3.86$ & 0.2 \\
\hline Model $2 \dagger$ & 1.00 & 2.03 & $0.92,4.45$ & 1.60 & $0.71,3.61$ & 0.3 \\
\hline Model 3ł & 1.00 & $2 \cdot 18$ & $0.95,5.00$ & 1.69 & $0.72,4.00$ & 0.3 \\
\hline \multicolumn{7}{|c|}{ Hypo-HDL-cholesterolaemia } \\
\hline Cases $(n)$ & 27 & & 28 & & 34 & \\
\hline Model 1 & 1.00 & 1.07 & $0.59,1.94$ & 1.31 & $0.74,2.32$ & 0.4 \\
\hline Model 2 & 1.00 & $1 \cdot 12$ & $0.61,2.06$ & 1.27 & $0.70,2 \cdot 28$ & 0.4 \\
\hline Model 3 & 1.00 & $1 \cdot 17$ & $0.63,2 \cdot 19$ & 1.36 & $0.74,2.49$ & $0 \cdot 3$ \\
\hline \multicolumn{7}{|c|}{ Hyper-LDL-cholesterolaemia } \\
\hline Cases $(n)$ & 12 & & 12 & & 12 & \\
\hline Model 1 & 1.00 & 1.07 & $0.46,2.49$ & $1 \cdot 10$ & $0.47,2.56$ & 0.8 \\
\hline Model 2 & 1.00 & 1.01 & $0.43,2.37$ & 1.04 & $0.44,2.44$ & 0.9 \\
\hline Model 3 & 1.00 & 0.95 & $0.39,2.35$ & 0.95 & $0.39,2.35$ & 0.9 \\
\hline \multicolumn{7}{|c|}{ Hypertriacylglycerolaemia } \\
\hline Cases $(n)$ & 34 & & 36 & & 29 & \\
\hline Model 1 & 1.00 & $1 \cdot 10$ & $0.64,1.91$ & 0.88 & $0.50,1.56$ & 0.7 \\
\hline Model 2 & 1.00 & $1 \cdot 12$ & $0.63,1.98$ & 0.84 & $0.47,1.53$ & 0.6 \\
\hline Model 3 & 1.00 & $1 \cdot 14$ & $0.63,2.04$ & 0.93 & $0.51,1.70$ & 0.8 \\
\hline \multicolumn{7}{|l|}{ Dyslipidaemia } \\
\hline Cases $(n)$ & 57 & & 65 & & 64 & \\
\hline Model 1 & 1.00 & $1 \cdot 29$ & $0.82,2.02$ & $1 \cdot 28$ & $0.81,2 \cdot 01$ & 0.3 \\
\hline Model 2 & 1.00 & 1.31 & $0.82,2 \cdot 10$ & 1.26 & $0.79,2.01$ & 0.3 \\
\hline Model 3 & 1.00 & $1 \cdot 37$ & $0 \cdot 84,2 \cdot 21$ & 1.34 & $0.83,2.18$ & 0.2 \\
\hline
\end{tabular}

such as lipid dysregulation ${ }^{(16,23,24,37,41-48)}$. However, the cumulative antioxidant levels, resulting from the prolonged and/or unnecessary intakes of dietary supplements, including fat-soluble antioxidants that are stored and not easily depleted in the body, may be high or even excessive among dietary supplement users. In addition, considering that the effects of antioxidants are synergistic, the net interactive antioxidant effect might be higher than the sum of the individual antioxidant effects ${ }^{(49)}$. Thus, it is plausible that high Se levels in dietary supplement users might contribute to high total antioxidant levels that may far exceed the upper limit of the biologically safe range for antioxidants. Furthermore, excessive levels of antioxidants in the body may inhibit normal oxidant-antioxidant defense functions that require optimal levels of free radicals for the tight regulation of metabolic pathways ${ }^{(38)}$, including prevention of lipid dysregulation.

Epidemiological studies investigating the association between dietary supplementation and serum lipid levels have shown inconsistent results. In the Supplementation en Vitamines et Mineraux Antioxydants (SU.VI.MAX) study, participants receiving long-term antioxidant supplements, including Se $(100 \mu \mathrm{g} / \mathrm{d})$, exhibited increased serum TAG levels compared with those receiving a placebo ${ }^{(50)}$. Similarly, a randomised study of a Chinese population showed that long-term use of supplements with vitamin $\mathrm{C}$, vitamin $\mathrm{E}$ and Se was associated 
Table 4. Lipid profiles and dyslipidaemia according to the tertiles $(T)$ of toenail selenium levels, stratified by dietary supplement use (Odds ratios and $95 \%$ confidence intervals)

\begin{tabular}{|c|c|c|c|c|c|c|}
\hline \multirow[b]{3}{*}{ Dietary supplement uses } & \multicolumn{5}{|c|}{ Tertile of toenail Se $(\mu \mathrm{g} / \mathrm{g})$} & \multirow[b]{3}{*}{$P_{\text {for trend }}$} \\
\hline & \multirow{2}{*}{$\frac{\mathrm{T} 1}{\mathrm{OR}}$} & \multicolumn{2}{|c|}{$\mathrm{T} 2$} & \multicolumn{2}{|c|}{ T3 } & \\
\hline & & OR & $95 \% \mathrm{Cl}$ & OR & $95 \% \mathrm{Cl}$ & \\
\hline \multicolumn{7}{|l|}{ Users } \\
\hline Hypercholesterolaemia & 1.00 & 1.86 & $0.56,6.18$ & 3.56 & $1 \cdot 12,11 \cdot 26$ & 0.03 \\
\hline Hyper-LDL-cholesterolaemia & 1.00 & 1.89 & $0.52,6.84$ & $2 \cdot 12$ & $0.56,8.02$ & 0.3 \\
\hline Hypo-HDL-cholesterolaemia & 1.00 & 1.48 & $0.63,3.46$ & 1.89 & $0.84,4.25$ & 0.1 \\
\hline Hypertriacylglycerolaemia & 1.00 & 1.65 & $0.65,4.19$ & $2 \cdot 68$ & $1.09,6.64$ & 0.03 \\
\hline Dyslipidaemia & 1.00 & 1.71 & $0.87,3.34$ & 2.72 & $1.41,5 \cdot 26$ & 0.003 \\
\hline \multicolumn{7}{|l|}{ Non-users } \\
\hline Hypercholesterolaemia & 1.00 & $2 \cdot 69$ & $0.78,9.33$ & 0.56 & $0.12,2.67$ & 0.5 \\
\hline Hyper-LDL-cholesterolaemia & 1.00 & 0.52 & $0.12,2.25$ & 0.70 & $0.16,3.02$ & 0.6 \\
\hline Hypo-HDL-cholesterolaemia & 1.00 & 0.81 & $0.29,2.24$ & 0.73 & $0.25,2.08$ & 0.5 \\
\hline Hypertriacylglycerolaemia & 1.00 & 0.72 & $0.31,1.68$ & 0.21 & $0.07,0.59$ & 0.003 \\
\hline Dyslipidaemia & 1.00 & 1.01 & $0.48,2.14$ & 0.40 & $0.17,0.91$ & 0.03 \\
\hline
\end{tabular}

with small, but significant, increases in TC and LDL-cholesterol levels ${ }^{(51)}$. Prior two large trials, the Se and Vitamin E Cancer Prevention Trial ${ }^{(52)}$ and the Nutritional Prevention of Cancer Trial $^{(53)}$, found that Se supplementation did not prevent type 2 diabetes. On the contrary, both the trials the latter study raised the concern that Se supplementation might increase the risk for type 2 diabetes. Furthermore, cumulative evidence has suggested that excessive intake of Se may result in hair loss, weak nails, lack of mental alertness, garlic breath odour and excessive tooth decay and discolouration ${ }^{(43,54)}$. In contrast to the aforementioned trials, observational studies conducted in Finland, China, the USA and the UK reported inconsistent results ${ }^{(17,19,55,56)}$.

Dietary supplement use has been a controversial topic in a number of studies ${ }^{(57-60)}$. First, dietary supplements can affect health in various ways depending on the individual's current nutritional status. Dietary supplements may be effective if an individual has a nutrient deficiency ${ }^{(1,60,61)}$. However, if an individual's body has adequate nutrient levels before supplementation, additional use of dietary supplements may lead to toxicity and negative health effects ${ }^{(1,6,25,61)}$. Second, the complex interactions between various phytochemicals and other macromolecules present in whole foods may affect the absorption and bioavailability of various nutrients, including antioxidants ${ }^{(62,63)}$. Purified nutrients present in dietary supplements, on the other hand, may have a limited capacity to replicate these complex interactions, and, therefore, may not have the same bioavailability as nutrients from whole foods ${ }^{(62,63)}$.

The present study has several limitations. The study participants were limited to residents of the Yeungnam area of South Korea; thus, we cannot generalise the results of our study to other populations. Furthermore, this study analysed the baseline data of the SELEN cohort in a cross-sectional manner. Thus, it is possible that limitations exist in the investigation of causality between the exposure and outcome. To minimise this bias, information on metabolic function biomarkers, such as WC and blood lipids, was collected at least 6 months after the completion of initial data collection on exposure variables (e.g. toenail specimen collection). Further, as toenail Se concentration reflects long-term exposure to Se of up to 1 year, the occurrence of reverse causation might have been minimised in this study. Finally, although the size of toenail specimens and the timing of specimen collection may contribute to differences in the observed toenail Se levels, we did not fully control for such factors in the present study.

In conclusion, our findings showed that, among middle-aged adults living in the Yeungnam area of South Korea, the association between toenail Se levels, serum lipid levels and dyslipidaemia was significantly modified by dietary supplementation. Higher toenail Se levels were associated with a higher prevalence of lipid dysregulation among dietary supplement users; however, among supplement non-users, higher toenail Se levels were associated with a lower prevalence of dyslipidaemia and hypertriacylglycerolaemia. Additional large-scale, prospective cohort studies should be conducted in the future to further evaluate the association between Se intake and metabolic health effects in light of increasing rates of dietary supplement use.

\section{Acknowledgements}

The authors thank Hyo-Jin Kim, Sle Koo and Sukyung Cho for their technical contribution to the SELEN study. In addition, the authors thank the University of Missouri Research Reactor staff for the neutron activation analysis of samples.

This research was supported by the Basic Science Research Program through the National Research Foundation of Korea (NRF) funded by the Ministry of Science, ICT \& Future Planning (NRF-2014R1A1A3049866). The funding sponsors had no role in the design of the study; in the collection, analyses or interpretation of data; in the writing of the manuscript; or in the decision to publish the results.

J. J. conducted the analysis and wrote the manuscript. J .S. M. performed the assays. J. S. M. and K. P. reviewed the manuscript. K. P. developed the study design, supervised the analysis and contributed to the discussion. All authors read and approved the final manuscript.

None of the authors has any conflicts of interest to declare. 


\section{References}

1. Rayman MP (2012) Selenium and human health. Lancet 379 , $1256-1268$.

2. Santhosh Kumar B \& Priyadarsini KI (2014) Selenium nutrition: how important is it? Biomed Prev Nutr 4, 333-341.

3. Kryukov GV, Castellano S, Novoselov SV, et al. (2003) Characterization of mammalian selenoproteomes. Science $\mathbf{3 0 0}$, 1439-1443.

4. Fulgoni VL 3rd, Chu Y, O'Shea M, et al. (2015) Oatmeal consumption is associated with better diet quality and lower body mass index in adults: the National Health and Nutrition Examination Survey (NHANES), 2001-2010. Nutr Res 35, 1052-1059.

5. Rose M, Baxter M, Brereton N, et al. (2010) Dietary exposure to metals and other elements in the 2006 UK Total Diet Study and some trends over the last 30 years. Food Addit Contam Part A Chem Anal Control Expo Risk Assess 27, 1380-1404.

6. Tinggi U (2008) Selenium: its role as antioxidant in human health. Environ Health Prev Med 13, 102-108.

7. Berendsen AA, van Lieshout LE, van den Heuvel EG, et al. (2016) Conventional foods, followed by dietary supplements and fortified foods, are the key sources of vitamin D, vitamin $\mathrm{B}_{6}$, and selenium intake in Dutch participants of the NU-AGE study. Nutr Res 36, 1171-1181.

8. Meltzer HM, Bibow K, Paulsen IT, et al. (1993) Different bioavailability in humans of wheat and fish selenium as measured by blood platelet response to increased dietary Se. Biol Trace Elem Res 36, 229-241.

9. Mahalingam TR, Vijayalakshmi S, Prabhu RK, et al. (1997) Studies on some trace and minor elements in blood. A survey of the Kalpakkam (India) population. Part III: studies on dietary intake and its correlation to blood levels. Biol Trace Elem Res 57, 223-238.

10. Tapiero H, Townsend DM \& Tew KD (2003) The antioxidant role of selenium and seleno-compounds. Biomed Pharmacother 57, 134-144.

11. Xun P, Bujnowski D, Liu K, et al. (2011) Distribution of toenail selenium levels in young adult Caucasians and African Americans in the United States: the CARDIA Trace Element Study. Environ Res 111, 514-519.

12. Chen J (2012) An original discovery: selenium deficiency and Keshan disease (an endemic heart disease). Asia Pac J Clin Nutr 21, 320-326.

13. Oropeza-Moe M, Wisloff H \& Bernhoft A (2015) Selenium deficiency associated porcine and human cardiomyopathies. J Trace Elem Med Biol 31, 148-156.

14. Dhingra S \& Bansal MP (2006) Attenuation of LDL receptor gene expression by selenium deficiency during hypercholesterolemia. Mol Cell Biochem 282, 75-82.

15. Steinbrenner H, Bilgic E, Alili L, et al. (2006) Selenoprotein P protects endothelial cells from oxidative damage by stimulation of glutathione peroxidase expression and activity. Free Radic Res 40, 936-943.

16. Zhao Z, Barcus M, Kim J, et al. (2016) High dietary selenium intake alters lipid metabolism and protein synthesis in liver and muscle of pigs. J Nutr 146, 1625-1633.

17. Stranges S, Laclaustra M, Ji C, et al. (2010) Higher selenium status is associated with adverse blood lipid profile in British adults. J Nutr 140, 81-87.

18. Laclaustra M, Stranges S, Navas-Acien A, et al. (2010) Serum selenium and serum lipids in US adults: National Health and Nutrition Examination Survey (NHANES) 2003-2004. Atherosclerosis 210, 643-648.

19. Stranges S, Tabak AG, Guallar E, et al. (2011) Selenium status and blood lipids: the cardiovascular risk in Young Finns study. J Intern Med 270, 469-477.
20. Hays SM, Macey K, Nong A, et al. (2014) Biomonitoring equivalents for selenium. Regul Toxicol Pharmacol 70, 333-339.

21. Ferguson LR \& Karunasinghe N (2011) Nutrigenetics, nutrigenomics, and selenium. Front Genet 2, 15.

22. Cardoso BR, Busse AL, Hare DJ, et al. (2016) Pro198Leu polymorphism affects the selenium status and GPx activity in response to Brazil nut intake. Food Funct 7, 825-833.

23. Mao S, Zhang A \& Huang S (2014) Selenium supplementation and the risk of type 2 diabetes mellitus: a meta-analysis of randomized controlled trials. Endocrine 47, 758-763.

24. Park K \& Seo E (2017) Toenail mercury and dyslipidemia: interaction with selenium. J Trace Elem Med Biol 39, 43-49.

25. Rayman MP (2000) The importance of selenium to human health. Lancet 356, 233-241.

26. Ainsworth BE, Haskell WL, Leon AS, et al. (1993) Compendium of physical activities: classification of energy costs of human physical activities. Med Sci Sports Exerc 25, 71-80.

27. Korean National Health Insurance Service Health Checkup (1999) Health Insurance Guide. http://www.nhis.or.kr/static/ $\mathrm{html} / \mathrm{wbd} / \mathrm{g} / \mathrm{a} / \mathrm{wbdga0606.html} \mathrm{(accessed} \mathrm{March} \mathrm{2016).}$

28. Niroumand $\mathrm{S}$, Khajedaluee $\mathrm{M}$, Khadem-Rezaiyan $\mathrm{M}$, et al. (2015) Atherogenic Index of Plasma (AIP): a marker of cardiovascular disease. Med J Islam Repub Iran 29, 240.

29. Talbert RL (2003) Role of the National Cholesterol Education Program Adult treatment panel III guidelines in managing dyslipidemia. Am J Health Syst Pharm 60, S3-S8; quiz S25.

30. Lee Y \& Park K (2016) Reproducibility and validity of a semi-quantitative FFQ for trace elements. Br J Nutr 116, 864-873.

31. Steven Morris J, Stampfer MJ \& Willett W (1983) Dietary selenium in humans toenails as an indicator. Biolo Trace Elem Res 5, 529-537.

32. Corella D \& Ordovas JM (2015) Biomarkers: background, classification and guidelines for applications in nutritional epidemiology. Nutr Hosp 31, Suppl. 3, 177-188.

33. Slotnick MJ \& Nriagu JO (2006) Validity of human nails as a biomarker of arsenic and selenium exposure: a review. Environ Res 102, 125-139.

34. Garland M, Morris JS, Rosner BA, et al. (1993) Toenail trace element levels as biomarkers: reproducibility over a 6-year period. Cancer Epidemiol Biomarkers Prev 2, 493-497.

35. Hunter DJ, Morris JS, Chute CG, et al. (1990) Predictors of selenium concentration in human toenails. Am J Epidemiol 132, 114-122.

36. Longnecker MP, Taylor PR, Levander OA, et al. (1991) Selenium in diet, blood, and toenails in relation to human health in a seleniferous area. Am J Clin Nutr 53, 1288-1294.

37. Su L, Gao S, Unverzagt FW, et al. (2015) Selenium level and dyslipidemia in rural elderly Chinese. PLOS ONE 10, e0136706.

38. Park K, Rimm EB, Siscovick DS, et al. (2012) Toenail selenium and incidence of type 2 diabetes in U.S. men and women. Diabetes Care 35, 1544-1551.

39. Maasland DH, Schouten LJ, Kremer B, et al. (2016) Toenail selenium status and risk of subtypes of head-neck cancer: The Netherlands Cohort Study. Eur J Cancer 60, 83-92.

40. Fan AM \& Kizer KW (1990) Selenium. Nutritional, toxicologic, and clinical aspects. West J Med 153, 160-167.

41. Kristal AR, Darke AK, Morris JS, et al. (2014) Baseline selenium status and effects of selenium and vitamin e supplementation on prostate cancer risk. J Natl Cancer Inst 106, djt456.

42. Fortmann SP, Burda BU, Senger CA, et al. (2013) Vitamin and mineral supplements in the primary prevention of cardiovascular disease and cancer: an updated systematic evidence 
review for the U.S. Preventive Services Task Force. Ann Intern Med 159, 824-834.

43. Rees K, Hartley L, Day C, et al. (2013) Selenium supplementation for the primary prevention of cardiovascular disease. Cochrane Database Syst Rev, issue 1, CD009671.

44. Yoshizawa K, Ascherio A, Morris JS, et al. (2003) Prospective study of selenium levels in toenails and risk of coronary heart disease in men. Am J Epidemiol 158, 852-860.

45. Neve J (1996) Selenium as a risk factor for cardiovascular diseases. J Cardiovasc Risk 3, 42-47.

46. Sattler W, Maiorino M \& Stocker R (1994) Reduction of HDL- and LDL-associated cholesterylester and phospholipid hydroperoxides by phospholipid hydroperoxide glutathione peroxidase and Ebselen (PZ 51). Arch Biochem Biophys 309, 214-221.

47. Spallholz JE, Boylan LM \& Larsen HS (1990) Advances in understanding selenium's role in the immune system. Ann NY Acad Sci 587, 123-139.

48. Juszczuk-Kubiak E, Bujko K, Cymer M, et al. (2016) Effect of inorganic dietary selenium supplementation on selenoprotein and lipid metabolism gene expression patterns in liver and loin muscle of growing lambs. Biol Trace Elem Res 172, 336-345.

49. Palozza P \& Krinsky NI (1992) $\beta$-Carotene and $\alpha$-tocopherol are synergistic antioxidants. Arch Biochem Biophys 297, 184-187.

50. Hercberg S, Bertrais S, Czernichow S, et al. (2005) Alterations of the lipid profile after 7.5 years of low-dose antioxidant supplementation in the SU.VI.MAX Study. Lipids 40, 335-342.

51. Zhang L, Gail MH, Wang YQ, et al. (2006) A randomized factorial study of the effects of long-term garlic and micronutrient supplementation and of 2-wk antibiotic treatment for Helicobacter pylori infection on serum cholesterol and lipoproteins. Am J Clin Nutr 84, 912-919.

52. Lippman SM, Klein EA, Goodman PJ, et al. (2009) Effect of selenium and vitamin $\mathrm{E}$ on risk of prostate cancer and other cancers: the Selenium and Vitamin E Cancer Prevention Trial (SELECT). JAMA 301, 39-51.

53. Stranges S, Marshall JR, Natarajan R, et al. (2007) Effects of long-term selenium supplementation on the incidence of type 2 diabetes: a randomized trial. Ann Intern Med 147, 217-223.

54. Pedrero Z \& Madrid Y (2009) Novel approaches for selenium speciation in foodstuffs and biological specimens: a review. Anal Chim Acta 634, 135-152.

55. Chen C, Jin Y, Unverzagt FW, et al. (2015) The association between selenium and lipid levels: a longitudinal study in rural elderly Chinese. Arch Gerontol Geriatr 60, 147-152.

56. Christensen K, Werner M \& Malecki K (2015) Serum selenium and lipid levels: associations observed in the National Health and Nutrition Examination Survey (NHANES) 2011-2012. Environ Res 140, 76-84.

57. Watkins ML, Erickson JD, Thun MJ, et al. (2000) Multivitamin use and mortality in a large prospective study. Am J Epidemiol 152, 149-162.

58. Pocobelli G, Kristal AR, Patterson RE, et al. (2010) Total mortality risk in relation to use of less-common dietary supplements. Am J Clin Nutr 91, 1791-1800.

59. Li Y, Huang T, Zheng Y, et al. (2016) Folic acid supplementation and the risk of cardiovascular diseases: a metaanalysis of randomized controlled trials. J Am Heart Assoc 5, e003768.

60. Silver HJ (2009) Oral strategies to supplement older adults' dietary intakes: comparing the evidence. Nutr Rev 67, 21-31.

61. Bjelakovic G, Nikolova D \& Gluud C (2013) Meta-regression analyses, meta-analyses, and trial sequential analyses of the effects of supplementation with beta-carotene, vitamin A, and vitamin $\mathrm{E}$ singly or in different combinations on all-cause mortality: do we have evidence for lack of harm? PLOS ONE $\mathbf{8}$, e74558.

62. Burton-Freeman B \& Sesso HD (2014) Whole food versus supplement: comparing the clinical evidence of tomato intake and lycopene supplementation on cardiovascular risk factors. Adv Nutr 5, 457-485.

63. Liu RH (2003) Health benefits of fruit and vegetables are from additive and synergistic combinations of phytochemicals. Am J Clin Nutr 78, 517s-520s. 